\title{
In situ ultrasonic monitoring of zeolite A crystallization from coal fly ash
}

Nicholas M. Musyoka $\underline{a}_{2} *$, Leslie F. Petrik $\underline{a}$, Eric Hums $\underline{b}$, Hasan Baser $\underline{\mathrm{c}}$, Wilhelm Schwieger $\underline{\mathrm{c}}$

a Environmental and Nano Science Research Group, Department of Chemistry, University of the Western Cape, Private Bag X17, Bellville 7535, South Africa

b Consulting Environmental Catalysis, P.O. Box 1848, 91008 Erlangen, Germany

c Institute of Chemical Reaction Engineering, University of Erlangen - Nürnberg, 91058 Erlangen, Germany

\begin{abstract}
In this study, high phase purity of zeolite A was prepared from coal fly ash precursors. The molar regime of both the clear solution extract and unseparated fly ash slurry was adjusted to achieve the right com- position for zeolite A crystallization. The formation process for zeolite A from coal fly ash precursors was monitored in detail using an in situ ultrasonic system and was complemented by use of ex situ techniques such as XRD, FTIR, SEM and FTIR. The findings from both the in situ ultrasonic monitoring process and ex situ techniques clearly contributed significantly in unmasking the formation process of zeolite A from coal fly ash compared to previous studies reported in the literature. The study also enriches the existing body of literature by deeply investigating the gel-solutioncrystal interactions starting from this complex feedstock. Comparable ultrasonic signals were generated when both clear and unseparated fly ash based precursor solutions were used during the zeolite synthesis process.
\end{abstract}

Keywords: In situ, Ultrasound, Coal fly ash, Zeolite A, Mechanism, Ageing process

\section{Introduction}

Zeolites have traditionally been synthesized from conventional aluminosilicate gels prepared from pure chemicals $[1,2]$. Over recent years, unconventional starting materials such as coal fly ash [2-8], metakaolin [9], cupola slag and aluminum sludge [10], municipal solid waste ash [11], chrysotile and rice husk [12] have also been used to synthesize zeolites. The synthesis of zeolite from coal fly ash was pioneered by Holler and Wirsching [13], and since then numerous researchers have followed in synthesizing different types of zeolites [5]. Conversion of coal fly ash into zeolites not only alleviates fly ash disposal problems and minimizes environmental impact but also generates marketable zeolitic products that could offset disposal costs [14]. Zeolite A, which is denoted by International Zeolite Association (IZA) code as Linde Type A (LTA) [15] has successfully been synthesized from coal fly ash 
$[3,4,17]$ and has industrial applications such as in separation, ion exchange, sequestration of $\mathrm{CO}_{2}$ and catalysis among many other applications $[1,16]$. The use of fly ash based zeolites in catalysis has mostly been hindered by the lack of purity but recent studies have shown that pure phase zeolite $\mathrm{A}[3,4,17]$ could act as an alternative to the commercially available catalysts based on zeolite A. For example, Hui and Chao [18] applied fly ash-derived zeolite A in catalytic combustion of methane.

One of the challenges facing most zeolite researchers is the lack of ability to rationally control the synthesis process due to the limited understanding of the zeolite formation mechanism. This challenge also extends to the optimization and scale up process. Sankar and Bras [19] suggested that rational design of the synthesis process can be achieved when the zeolite crystallization mechanism is well understood. A deeper understanding of the formation mechanism will not only help in controlling and predicting the best conditions for synthesis but will also unmask the cooperative phenomena dictating chemical-structure-physical property relationships. Many attempts to understand the zeolite formation processes have been based on the use of ex situ monitoring techniques [20,22]. These techniques rely on periodic quenching of the reaction mixture, i.e. separating the solid and liquid phase prior to independent analysis. The challenge with this approach is that it is not so reliable since there could be artifacts caused by the quenching process. Recently, in situ monitoring methods have been applied by different researchers [20-22] and most of these developments are described in a recent review by Pienack and Bensch [23] who have classified the different in situ methods as; (i) in situ scattering techniques, (ii) in situ spectroscopy, (iii) in situ mass spectrometry, (iv) in situ transmission electron microscopy and (v) combination methods. In addition to the list, the in situ ultrasonic technique reported first by Toufar [24] and Schmachtl et al. [25] has also elicited great interest to contribute to the understanding of the complexity of the zeolite formation process. The in situ ultra- sonic monitoring technique is an indirect, non-invasive, phase insensitive method that is based on investigating the degree of interaction of ultrasonic wave transport properties with the zeolitic precursor species as it travels through the synthesis mixture.

The use of in situ ultrasound diagnosis of zeolite crystallization had been reported [25-27] to successfully enable monitoring of the complex formation process of zeolites from pure analytical grade sources of $\mathrm{Si}$ and Al. The use of ultrasonic attenuation as an investigation tool [28] was an improvement of the earlier use of ultrasound velocity and amplitude [24-27] since there were challenges encountered due to scattering from the solid products which made it difficult to correlate ultrasound phase and amplitude with the increasing zeolites crystallinity during the crystal growth step [28]. In the current study, zeolite A prepared from fly ash was chosen to act as a model for investigating the crystallization mechanism of zeolites from fly ash because it is well understood and a lot of effort has already been made to understand its formation process from pure analytical grade sources aluminate 
and silica [29]. In addition, this zeolite crystallizes under reasonable hydrother mal conditions and short synthesis times. Since no single technique can be suitable to fully elucidate the key steps of zeolite crystallization, other ex situ techniques such as X-ray diffraction (XRD), Scanning Electron Microscopy (SEM), Fourier Transform Infrared (FTIR) spectroscopy and Inductively Coupled Plasma Atomic Emission Spectrometry (ICP-AES) were employed to complement the in situ ultrasonic monitoring process, to confirm the results and support the findings for the entire crystallization process.

\section{Experimental}

\subsection{Zeolite synthesis procedure}

Zeolite A was synthesized from a precursor solution extracted from South African class $\mathrm{F}$ coal fly ash that had a molar ratio of $1 \mathrm{Al}_{2} \mathrm{O}_{3}: 31 \mathrm{Na}_{2} \mathrm{O}: 4 \mathrm{SiO}_{2}: 415 \mathrm{H}_{2} \mathrm{O}$. This was achieved by initially fusing the fly ash with sodium hydroxide in the ratio of 1:2 at $550{ }^{\circ} \mathrm{C}$ for $1.5 \mathrm{~h}$ to convert the insoluble fly ash mineral phase to soluble sodium aluminosilicate phase. The resulting solid material was cooled down and dissolved in demineralized water in a ratio of 1:5, stirred for $2 \mathrm{~h}$, and then the solid and liquid phases of the slurry were separated by filtration after centrifuging. The clear solution $(72 \mathrm{ml})$ recovered after filtration was used as the source of $\mathrm{Al}$ and Si during the ageing and hydrothermal synthesis process. On a different experimental run, the unseparated fly ash slurry was also used as precursor. In order to achieve the specified molar composition for synthesis of zeolite A, extra aluminate solution was added. The aluminate solution $(28 \mathrm{ml})$ was prepared by mixing (1.38 g) commercial sodium aluminate solid (Riedel-de Haën) and (2.78 g) sodium hydroxide (Merck) in water $(28 \mathrm{ml})$. The resulting precursor mixtures $(100 \mathrm{ml})$ of either clear solution or unseparated fly ash slurry were transferred into the in situ ultrasonic monitoring glass container. To study the effect of ageing of the precursor species, the reaction mixture was placed in the in situ ultrasonic monitoring glass container to age at room temperature for different times (360, 720 and $1050 \mathrm{~min}$ ) before heating was commenced. The heating rate was around $0.5{ }^{\circ} \mathrm{C} / \mathrm{min}$ and the final reaction temperature for hydrothermal synthesis was set at $80{ }^{\circ} \mathrm{C}$ for $360 \mathrm{~min}$. In a separate experiment, the fly ash based molar composition of the synthesis mixture was simulated using pure analytical grade sources of silica (water glass $\left(28 \% \mathrm{SiO}_{2}, 27 \%\right.$ $\mathrm{Na} 2 \mathrm{O}$, Riedel-de Haën), sodium aluminate powder $\left(\mathrm{NaAlO}_{2}, 28 \% \mathrm{Al}_{2} \mathrm{O}_{3}, 27 \% \mathrm{Na} 2 \mathrm{O}\right.$, Riedel-de Haën) and sodium hydroxide (>99\% NaOH; Merck).

\subsection{In situ ultrasonic monitoring set-up and methodology}

A schematic of the in situ ultrasonic monitoring set-up is shown in Fig. 1. The glass container had an ultrasonic transducer $(10 \mathrm{~mm}$ 


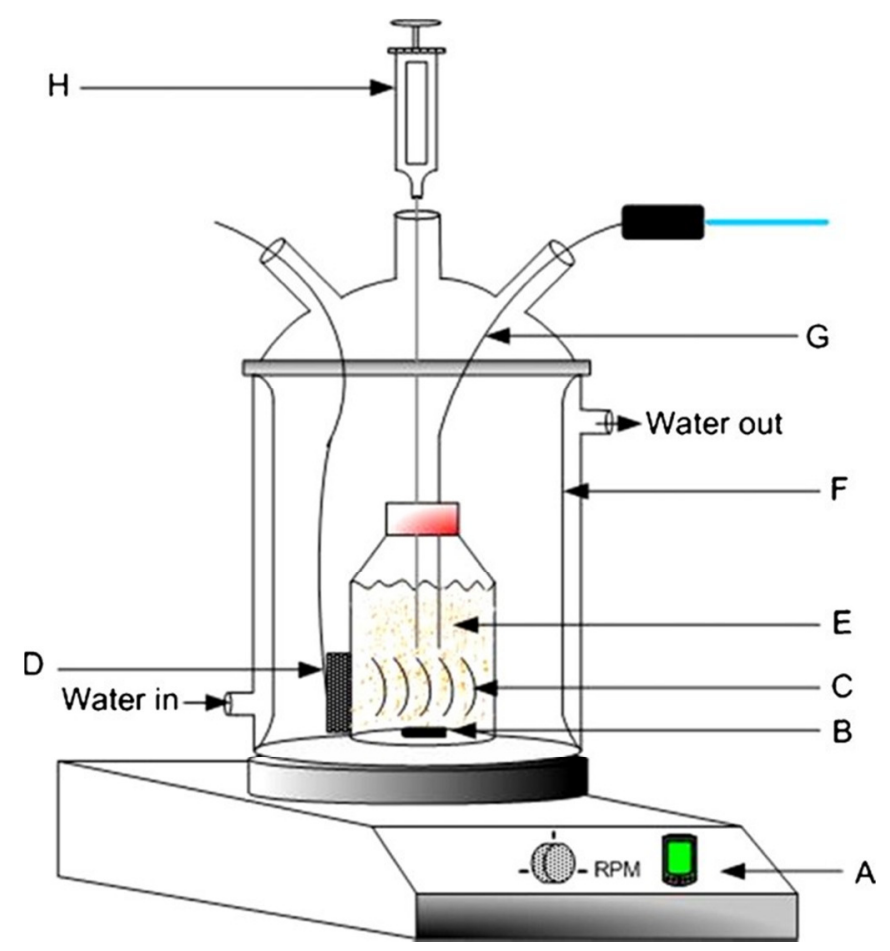

Fig. 1. Schematic of the in situ ultrasonic monitoring set-up; magnetic stirrer (A), magnetic stirring rod (B), ultrasonic waves (C), ultrasonic transducer (D) synthesis mixture (E), thermostated double walled container (F), thermocouple $(\mathrm{G})$, and syringe $(\mathrm{H})$.

Ø, PIC 155, central frequency: $2 \mathrm{MHz}$, supplied by PI Ceramic, Germany) attached on the outer wall of the glass container and was able to transmit and receive ultrasound waves via the pulse-echo method. The ultrasonic signal pathway was 52 $\mathrm{mm}$. The smooth surface of the glass, opposite the side to which the transducer was attached, acted as the reflecting surface. A syringe was inserted in the glass container and the tip was about $3 \mathrm{~cm}$ above the pathway of the ultrasound to enable collection of samples during the in situ monitoring process. A thermocouple was also attached to the glass container for online temperature monitoring. The ultrasonic glass container was transferred into a double-walled glass reactor which was heated by circulating water. The precursor mixture was stirred during the entire in situ monitoring process to enhance homogenization and avoid sedimentation. Attenuation of the ultrasound signal travelling through the precursor mixture during both the ageing and hydrothermal synthesis was monitored and the data was captured by PBP Optel software (Ver. 4.1/2004) using a Opbox 01/100 manufactured by Optel Ltd. Changes of ultrasonic attenuation were recorded after every $1 \mathrm{~min}$. Initially, independent blank in situ ultrasonic monitoring runs of demineralized water and $\mathrm{NaOH}(6 \mathrm{M})$ solution were conducted to investigate the effect ultrasound wave properties on heating the blank solutions. 


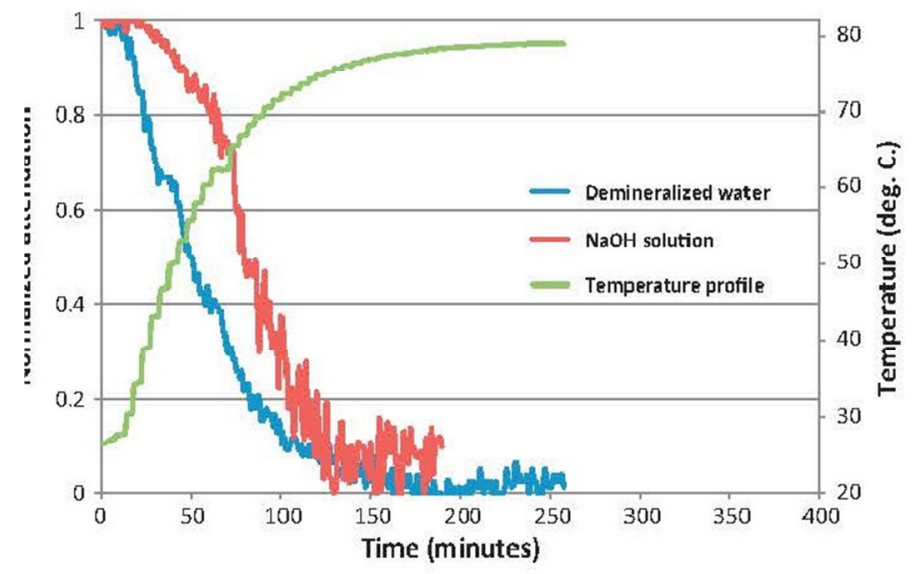

ig.2. Plots of normalized attenuationvs. time ofblank runs of demineralized water

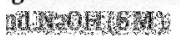

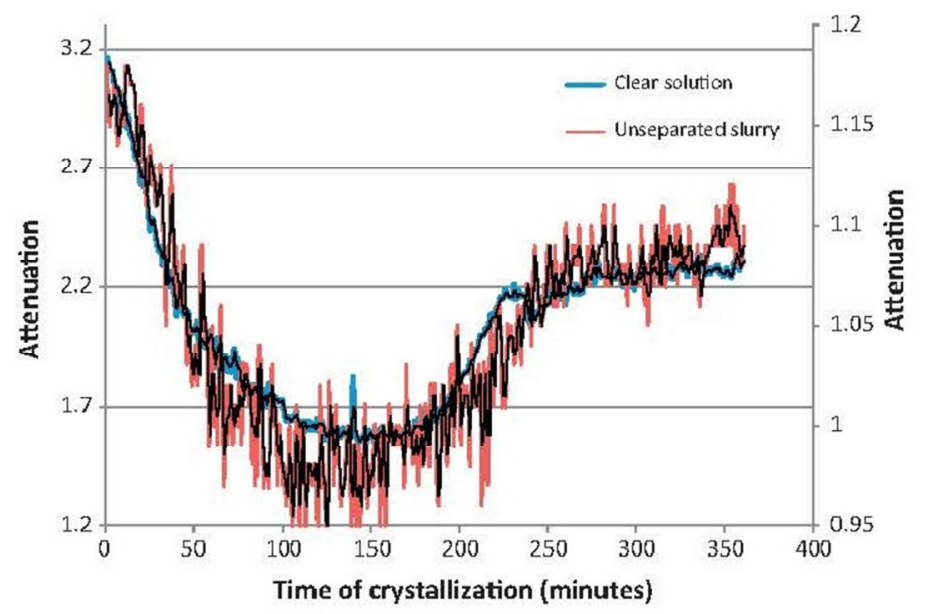

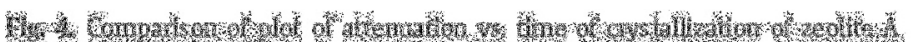

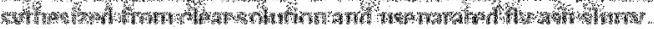

Fig. 2. Plots of normalized attenuation vs. time of blank runs of demineralized water and $\mathrm{NaOH}(6 \mathrm{M})$.

Time of crystallization (minutes)

Fig. 4. Comparison of plot of attenuation vs. time of crystallization of zeolite A sythesized from clear solution and useparated fly ash slurry.

\subsection{Complementary ex situ monitoring of zeolite formation process}

Small aliquots of samples $(4 \mathrm{ml})$ were extracted from the reac- tion mixture at predetermined times (o, 30, 60, 90, 120, 150, 200, 210, 220, 240 and $360 \mathrm{~min}$ ) without disturbing the in situ ultrasonic monitoring process. In each case the solid and liquid phase was separated by filtration after centrifuging the sample. The recovered solid samples were further washed using demineralized water and dried overnight at $110{ }^{\circ} \mathrm{C}$ prior to ex situ analysis. The concentration of $\mathrm{Si}$ and $\mathrm{Al}$ in the liquid phase was determined using Inductively Coupled Plasma Atomic Emission Spectrometry

\section{Clear solution Unseparated slurry}

(ICP-AES, Perkin-Elmer). Crystallinity of the solid samples was mea- sured using a Philips X-pert pro MPD X-ray diffractometer and the samples were scanned over a range of $4-60^{\circ}$ 2e. Crystalline phases were identified with the help of Highscore Xpert software. Mor- phological analysis of the solid samples was conducted using a Scanning Electron Microscope (Gemini). Structural analysis of the solid samples was conducted using a Fourier Transform Infrared spectrometer (JASCO FT/IR-4100).

\section{Results}




\subsection{Measurements of attenuated ultrasound signal}

Fig. 2 presents results of in situ ultrasonic monitoring of blank demineralized water and $\mathrm{NaOH}(6 \mathrm{M})$ solution which showed a reduction of ultrasonic attenuation for the first $120 \mathrm{~min}$ before stabilization. These blank experiments enabled decoupling of interferences that might inhibit analysis of reactions taking place in the actual synthesis mixture presented in Fig. 3. In the initial $30 \mathrm{~min}$ of in situ ultrasonic monitoring of the synthesis mixture, the ultrasonic attenuated signal was observed to decrease but as the synthesis time progressed there was a slight increase that occurred between 50 and 80 min. Thereafter, a decrease of US-attenuation up to about after 140 min was observed. Between 140 and $180 \mathrm{~min}$, the attenuated signal was noted to stabilize without any noticeable increase or decrease but increased steadily between 180 and $220 \mathrm{~min}$. A sharp inflection point was noted at $220 \mathrm{~min}$ and was followed by a dramatic increase of the ultrasonic attenuation that occurred between 240 and 260 min before it stabilized until the end of the monitoring process. To understand the differences of processes taking place during the hydrothermal synthesis of zeolite A from clear solution and unseparated fly ash slurry, Fig. 4 presents results that compare the in situ ultrasonic signals generated when these two different precursor mixtures were investigated at $80^{\circ} \mathrm{C}$.

\subsubsection{Effects of ageing}

Ageing studies have been widely used to generate indirect experimental data to understand the processes taking place in the early stages of crystallization of zeolites. Fig. 5 reports the effect of the ageing process as monitored by the in situ ultrasonic technique. When comparing the US-attenuation signals generated from synthesis conducted after ageing the reaction mixture at room temperature for the four different times, three characteristic effects could be observed. These ageingtime dependent influences were; (i) shortening of nucleation period, (ii) eradication of secondary amorphous phase formation step and (iii) disappearance of the 'hump' in US-attenuation pattern which signaled the absence of gel breakup step.

\subsubsection{Effects of simulating fly ash molar regime}

Fig. 6 compares the in situ attenuated ultrasonic signal generated when studying the hydrothermal synthesis of the fly ash based 


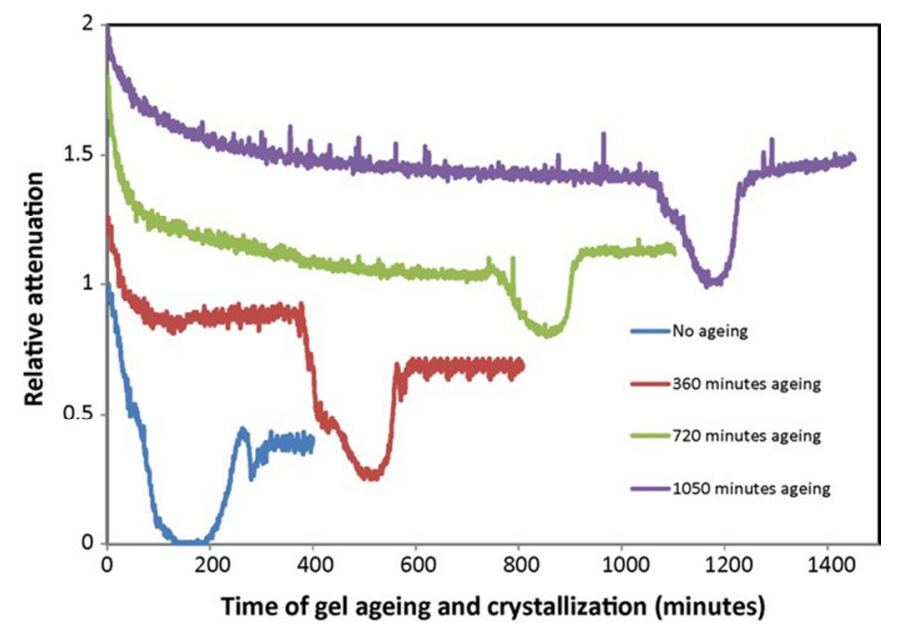

Time of crystallization (minutes)

Fig. 3. Plots of normalized attenuation vs. time of crystallization of zeolite A from clear solution (reproducibility tests). 


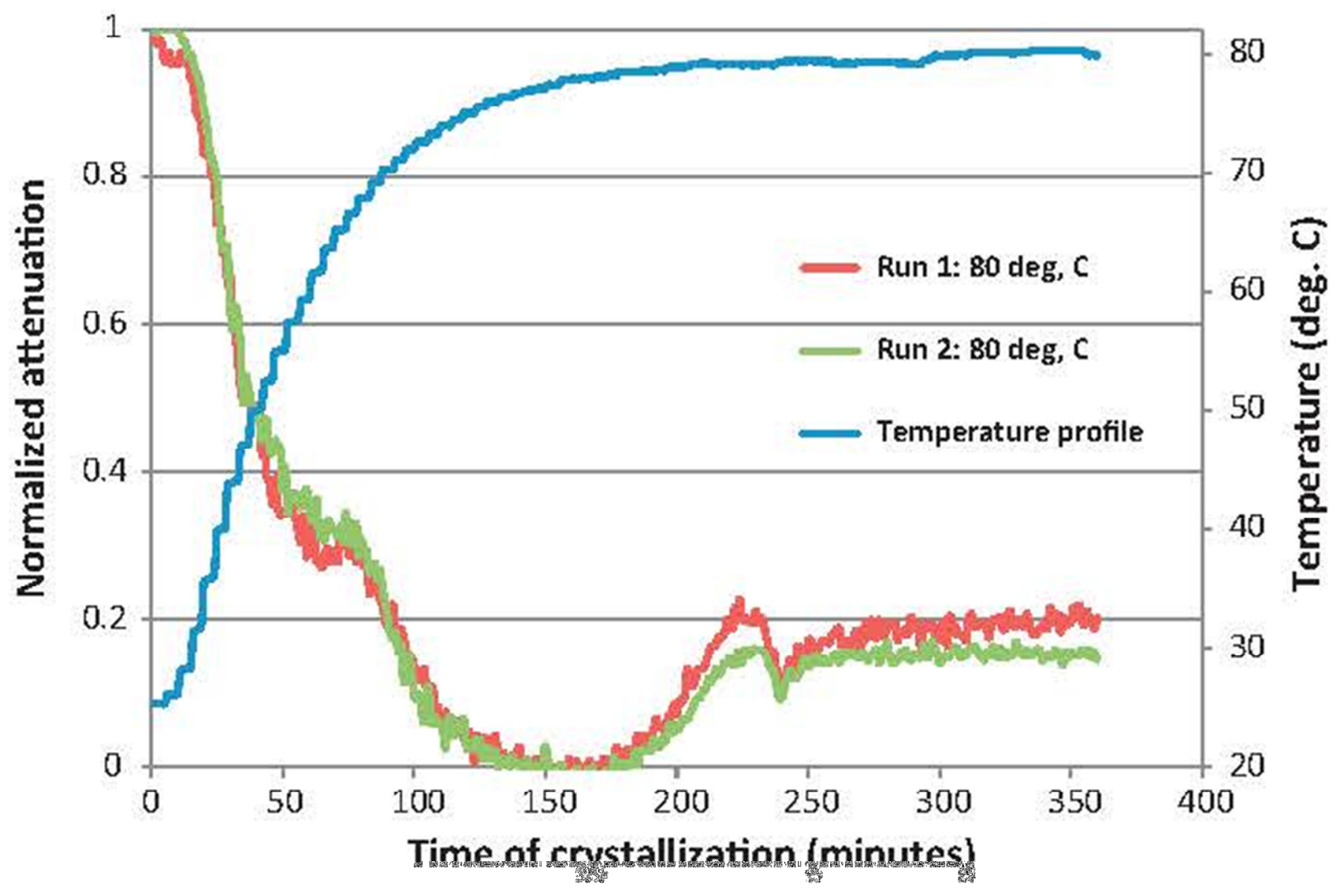

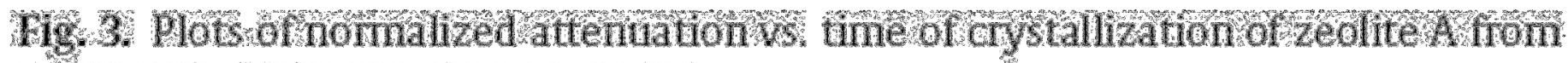
Clearsolution (teproducbilify tests

Fig. 5. Plots of attenuation vs. time of crystallization of zeolite A: effect of gel ageing before crystallization of zeolite $\mathrm{A}$ from clear solution at $80^{\circ} \mathrm{C}$ (the start point of the heating up is marked with an arrow). 


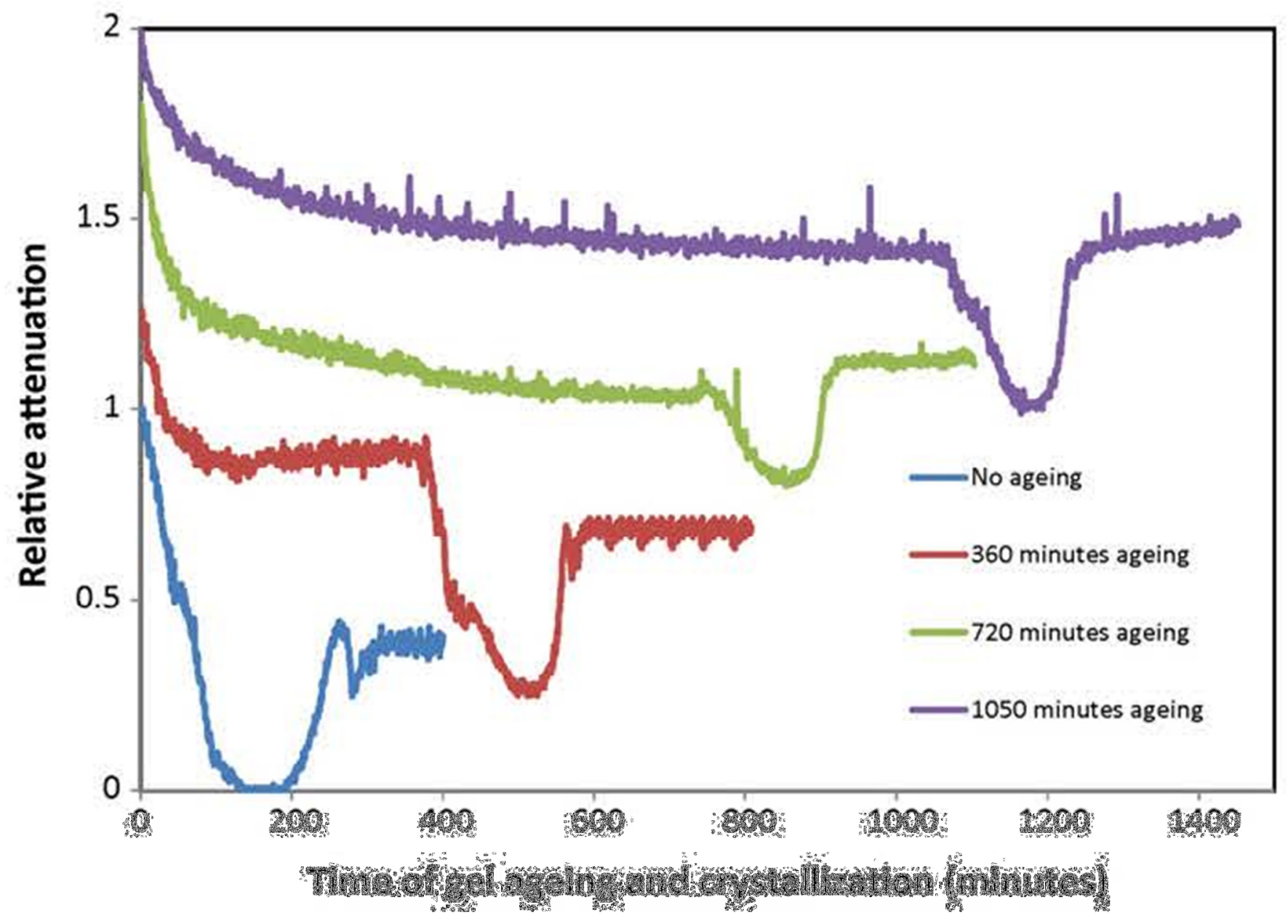

Fig. 5. Plots of attenuation vs. time of crystallization of zeolite A: effect of gel ageing before cructallization, of zeolite. Afrom clear solution at $80^{\circ} \mathrm{C}$ (thestartpoint of the Thain

zeolite A with that of the simulated molar composition generated using $\mathrm{Si}, \mathrm{Al}$ and $\mathrm{Na}$ derived from pure analytical grade commercial chemicals as described in Section 2.1. The immediate cloudiness of the precursor solution that had been observed after preparing the fly ash based precursor gel was not seen after mixing pure analytical grade aluminate and silicate chemicals but upon further the precursor mixture became cloudy. This naked-eye observation was also confirmed by the USattenuation signal which could be seen as a peak between 60 and 80 min in the ultrasonic attenuation pattern when the temperature was about $70{ }^{\circ} \mathrm{C}$. The hump in the during the study of fly ash based precursor gel was not observed in the attenuated pattern of the simulated composition.

\subsection{Complementary ex situ analyses}

3.2.1 XRD

Fig. 7 presents results of XRD analysis of solid samples that were synthesized from clear solution. Since the synthesis product extracted during the time dependent in 
situ monitoring study $(4 \mathrm{ml})$ was not enough for XRD analysis, independent runs corresponding to the respective extraction times (60, 120, 220 and $360 \mathrm{~min}$ ) were conducted using the in situ monitoring set-up and were used to track the progress of fly ash zeolitization. A broad hump in the XRD patterns, observed between $20^{\circ}$ and $40^{\circ} 2 \mathrm{e}$, appeared during the initial stages of the zeolite formation process (6o and $120 \mathrm{~min}$ ) but after $220 \mathrm{~min}$ the characteristic diffraction peaks of zeolite A were noted to appear and the reflections were observed to grow as time progresses up to 360 min. Data in Fig. 7 clearly highlights the disadvantages of ex situ XRD investigation due to the fact that fewer data points could be obtained which limits the extent of detailed information compared to results obtained from in situ ultrasonic analysis.

\subsubsection{SEM analysis}

As visibly observed and earlier reported [29], the $\mathrm{Si}$ and $\mathrm{Al}$ species were mostly present as a precipitated solid amorphous gel phase in the extracted samples $(0,30$, $60,90,120,150,200,210,220,240$ and $360 \mathrm{~min}$ ) while the balance of species which are present in the liquid phase exists as low molecular weight monomeric and oligomeric species. Upon successful separation of the solid and liquid phases, the crystallization process was indirectly reflected in the changes that had happened in the solid phase as examined using a Scanning Electron Microscope. Results of SEM analysis for the samples taken after the predetermined times are shown in Fig. 8. The relationship between gel dissolution process and zeolite A crystal growth is well captured in SEM micrographs obtained for samples extracted between 150 and 280 min. During these early stages of crystal growth, the crystal morphology is not well defined and the typical morphology (chamfered-edged) of zeolite A is only obtained after $280 \mathrm{~min}$. This shape remained distinct until when the reaction was terminated after $360 \mathrm{~min}$ at $80{ }^{\circ} \mathrm{C}$. Fig. 9(a) and (b) compares the morphology of zeolite A synthesized using a clear precursor solution with that from unseparated fly ash slurry. It was found that zeolite A crystals prepared from unseparated precursor slurry had sharp edged cornered morphology which was different from the chamfered edged crystals obtained from the clear solution. 


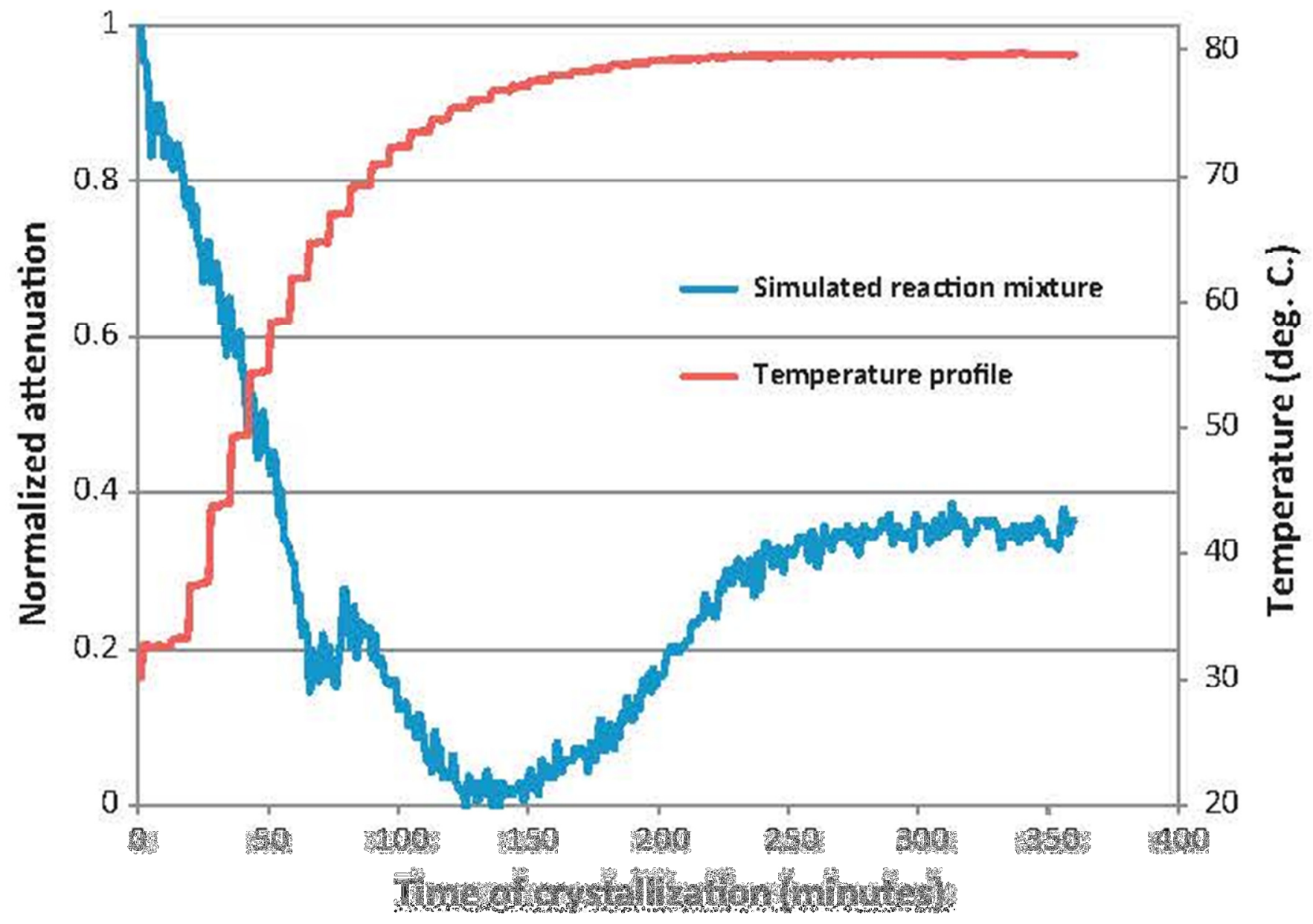

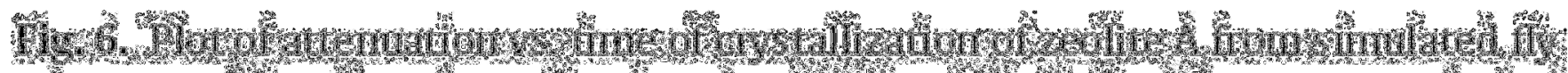

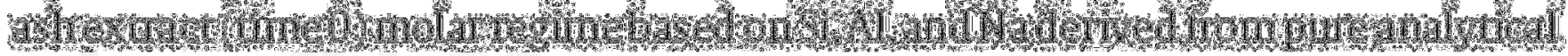
grade commercial chemicals.

Time of crystallization (minutes)

Fig. 6. Plot of attenuation vs. time of crystallization of zeolite A from simulated fly ash extract (time o) molar regime based on $\mathrm{Si}, \mathrm{Al}$, and $\mathrm{Na}$ derived from pure analytical grade commercial chemicals. 


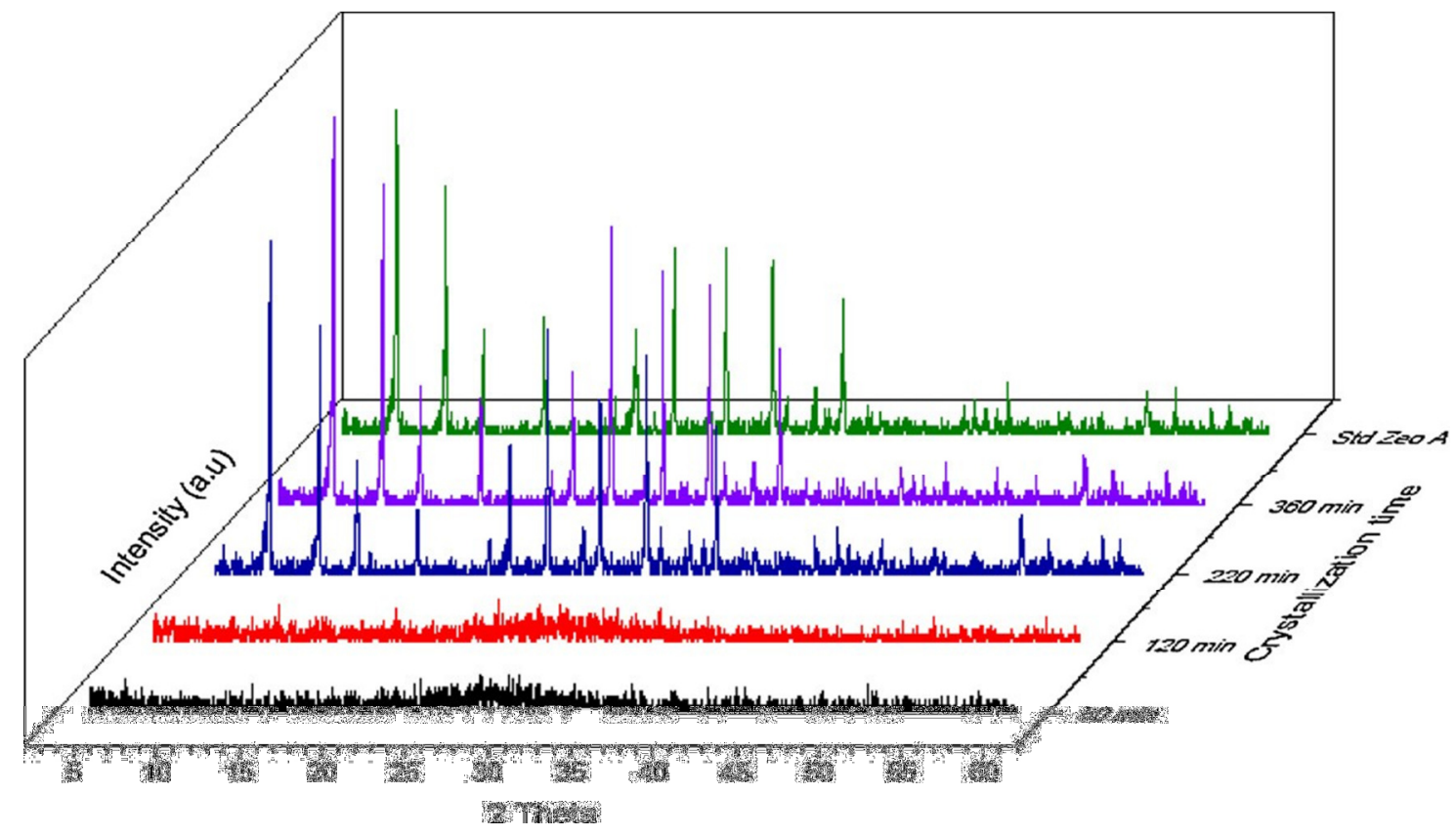

Fig. 7. XRD pattern of synthesis products obtained at different times corresponding with the in situ ultrasonic monitoring process with reference to standard zeolite A.
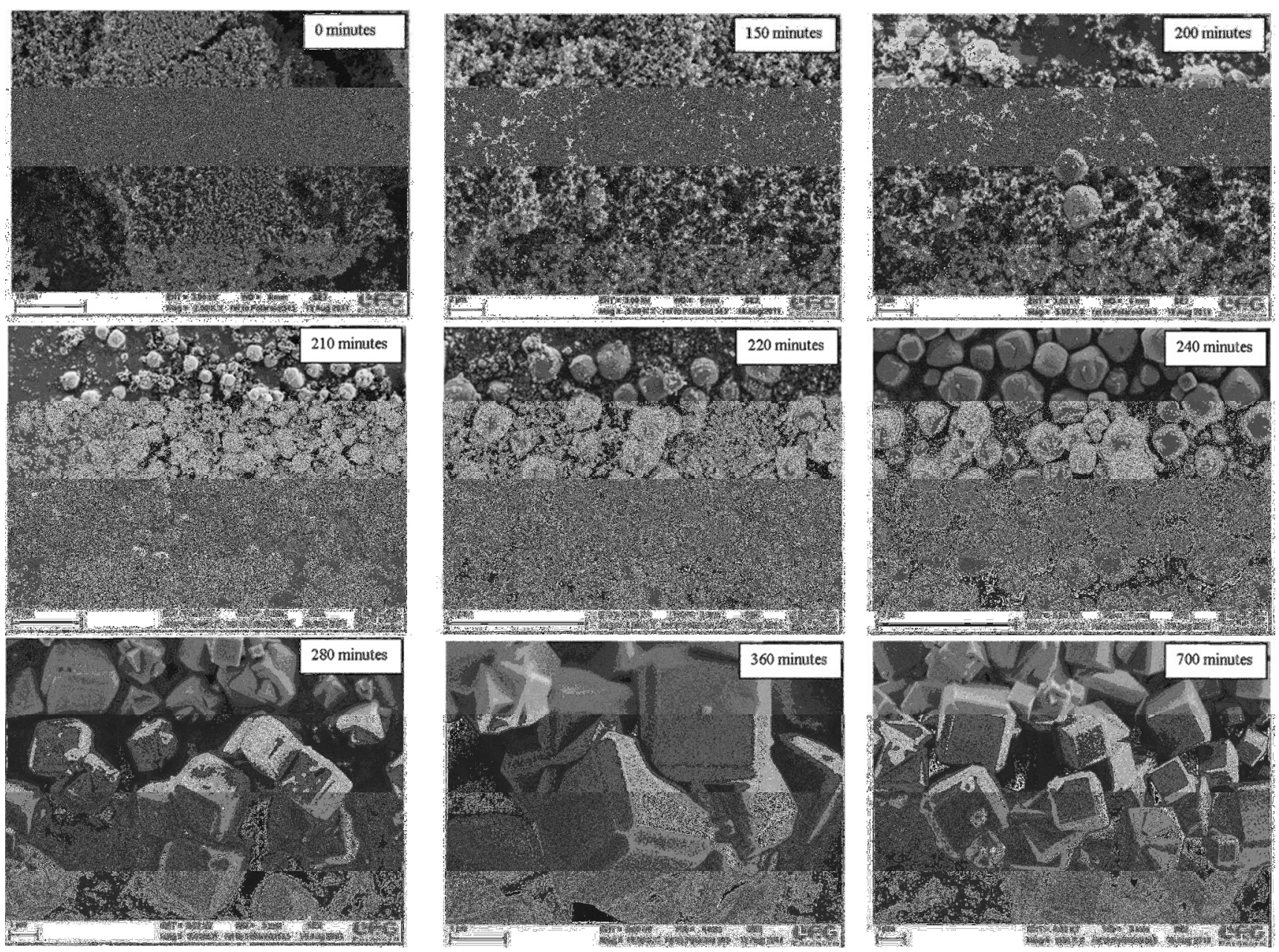
Fig. 8. SEM images of solid samples taken at different times during the in situ monitoring of crystallization process of zeolite A from clear solution.

\section{ICP analysis}

The slurry which was extracted from the synthesis mixture following the procedure referred in Section 2.3 was separated to get supernatant liquid and solid phase. Figs. 10 and 11 present the ICP results for changes of concentration of $\mathrm{Al}$ and Si obtained from chemical analysis of the supernatant solution which acted as an indirect way of monitoring the progress of zeolite formation. Analysis of the supernatant solution was preferred because it would minimize errors that could have otherwise have arisen from total digestion of the solid product. Both figures indicate the indirect connection between the molar composition of the reaction mixture and the rate of crystal growth. The concentration of $\mathrm{Al}$ and $\mathrm{Si}$ in the supernatant liquid was observed to decrease after the initial 30 min but increased slightly between 60 and $90 \mathrm{~min}$. Thereafter, the con- centration of the $\mathrm{Al}$ and $\mathrm{Si}$ in the solution was noted to show small fluctuations up to about $150 \mathrm{~min}$. A steady decrease in the concentration of $\mathrm{Si}$ and $\mathrm{Al}$ was noted to occur between 150 and 210 min with an inflection point occurring at around $220 \mathrm{~min}$ which was later proceeded by a steady decrease occurring between 220 and $360 \mathrm{~min}$. The trend of $\mathrm{Si} / \mathrm{Al}$ ratio shown in Fig. 12 demonstrates that the amount of free $\mathrm{Al}$ released after the break-down of the gel structure was higher than that Si.
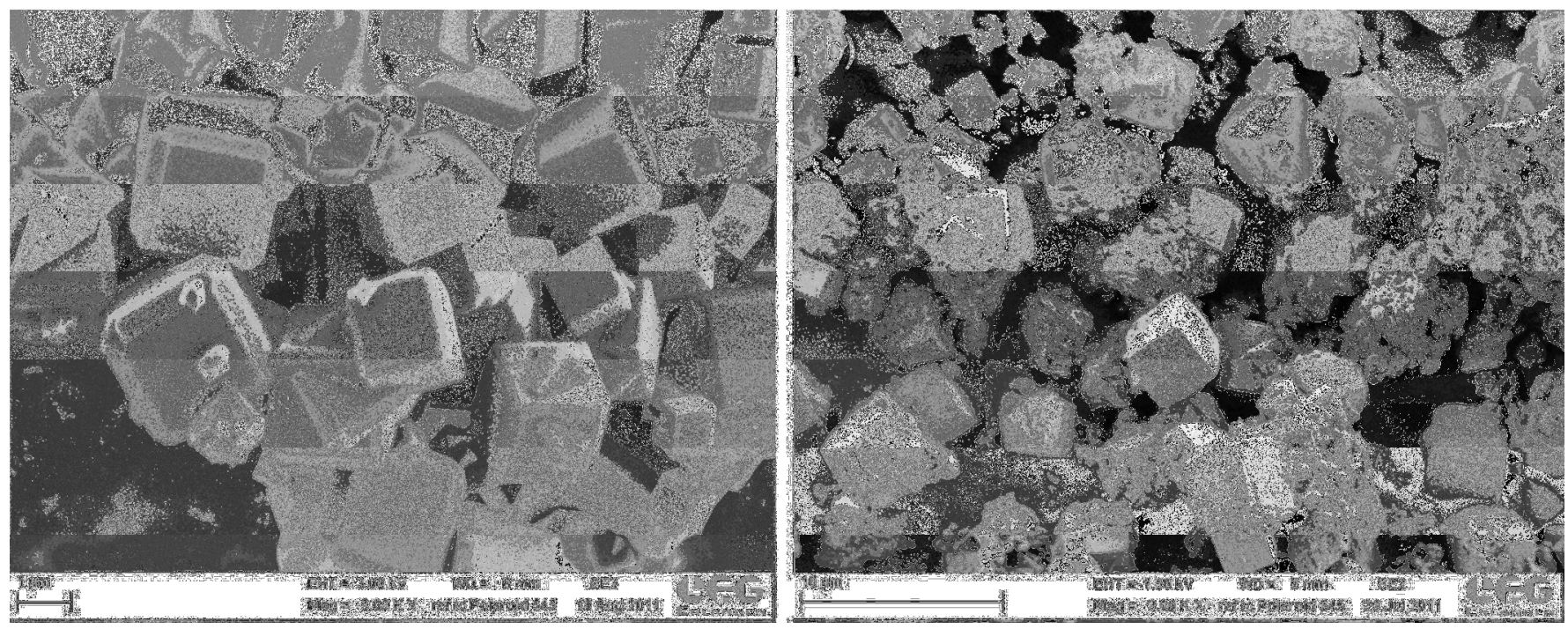

Fig. 9. SEM images of zeolite A samples from (a) clear solution and (b) unseparated fly ash slurry. 


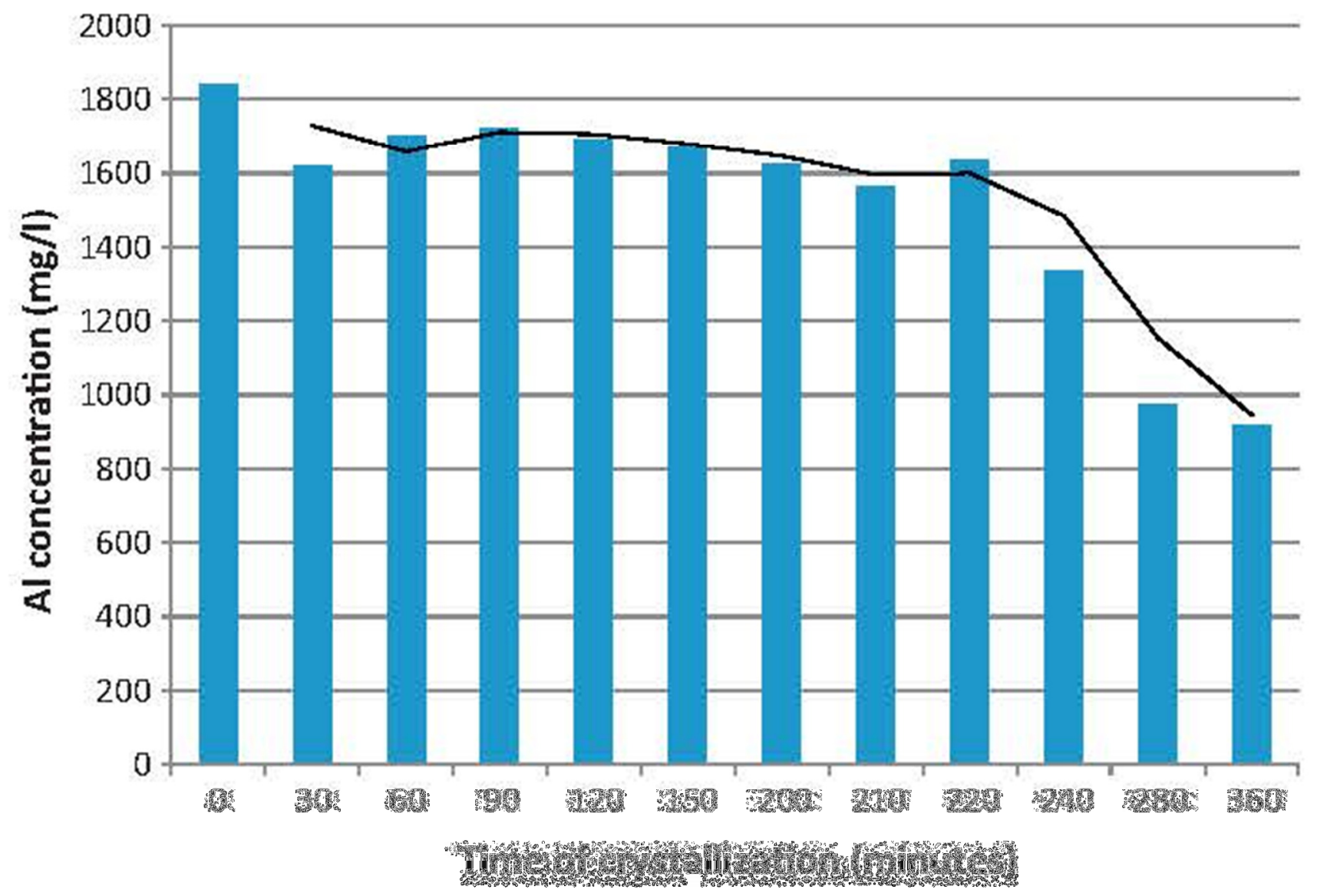

Fig. 10. ICP analysis: concentration of Al in the supernatant solution for samples taken at different times during in situ monitoring of the crystallization process of zeolite arrom dear solution

\subsubsection{FTIR analysis}

IR spectroscopy has been extensively used to monitor the development of crystallinity during zeolite synthesis [31]. Fig. 13 compares the infra-red spectra of synthesized zeolite A with its amorphous precursors and intermediates from samples extracted after different synthesis times (o, 30, 60, 90, 120, 150, 200, 210, 220,240 and $360 \mathrm{~min}$ ) during the in situ ultrasonic monitoring process 


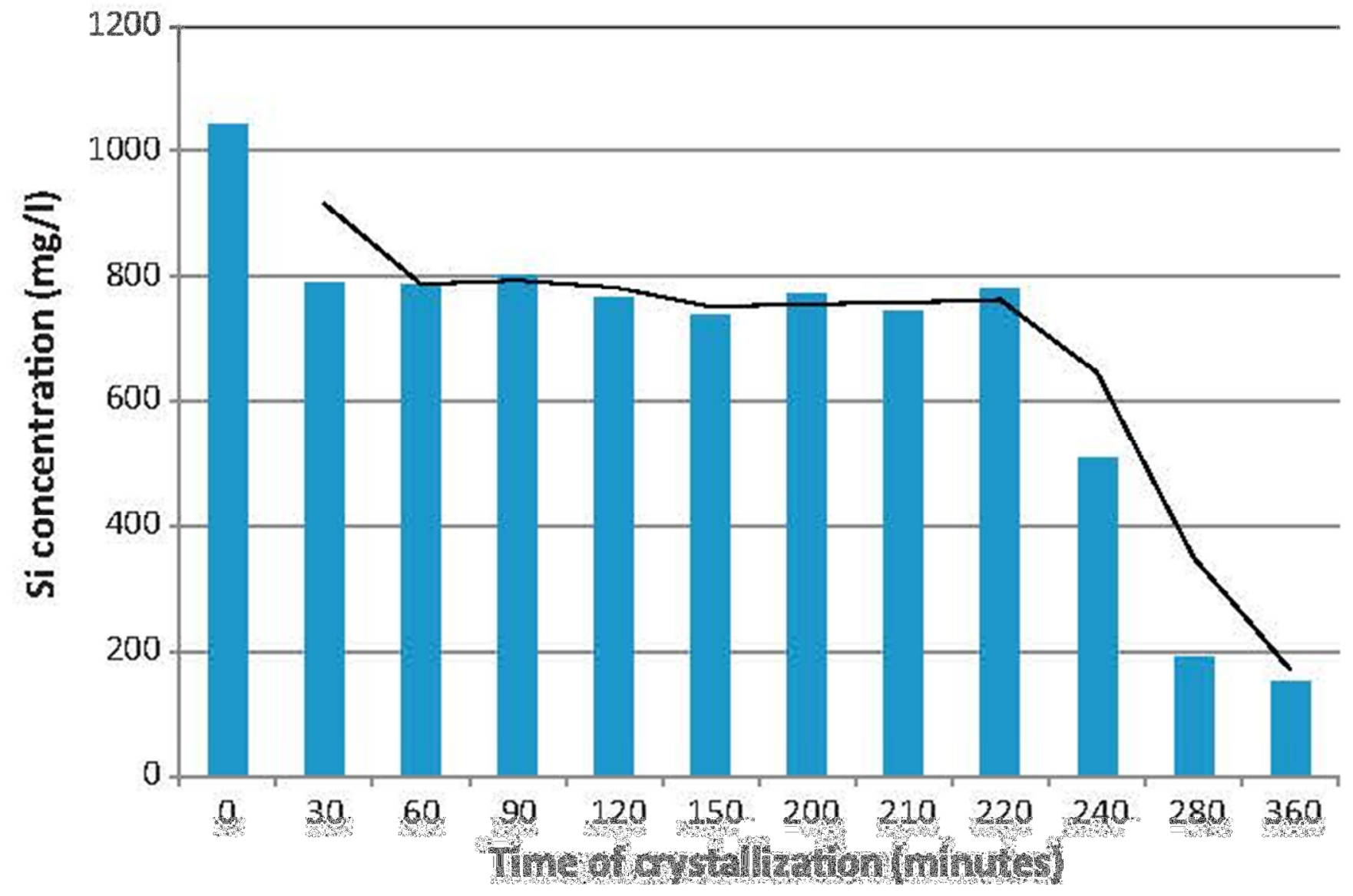

Fig. 11. ICP analysis: concentration of Si in the supernatant solution for samples taken at different times furing in situ monitoring of the crystallization process of zeolite A from dear solution: 


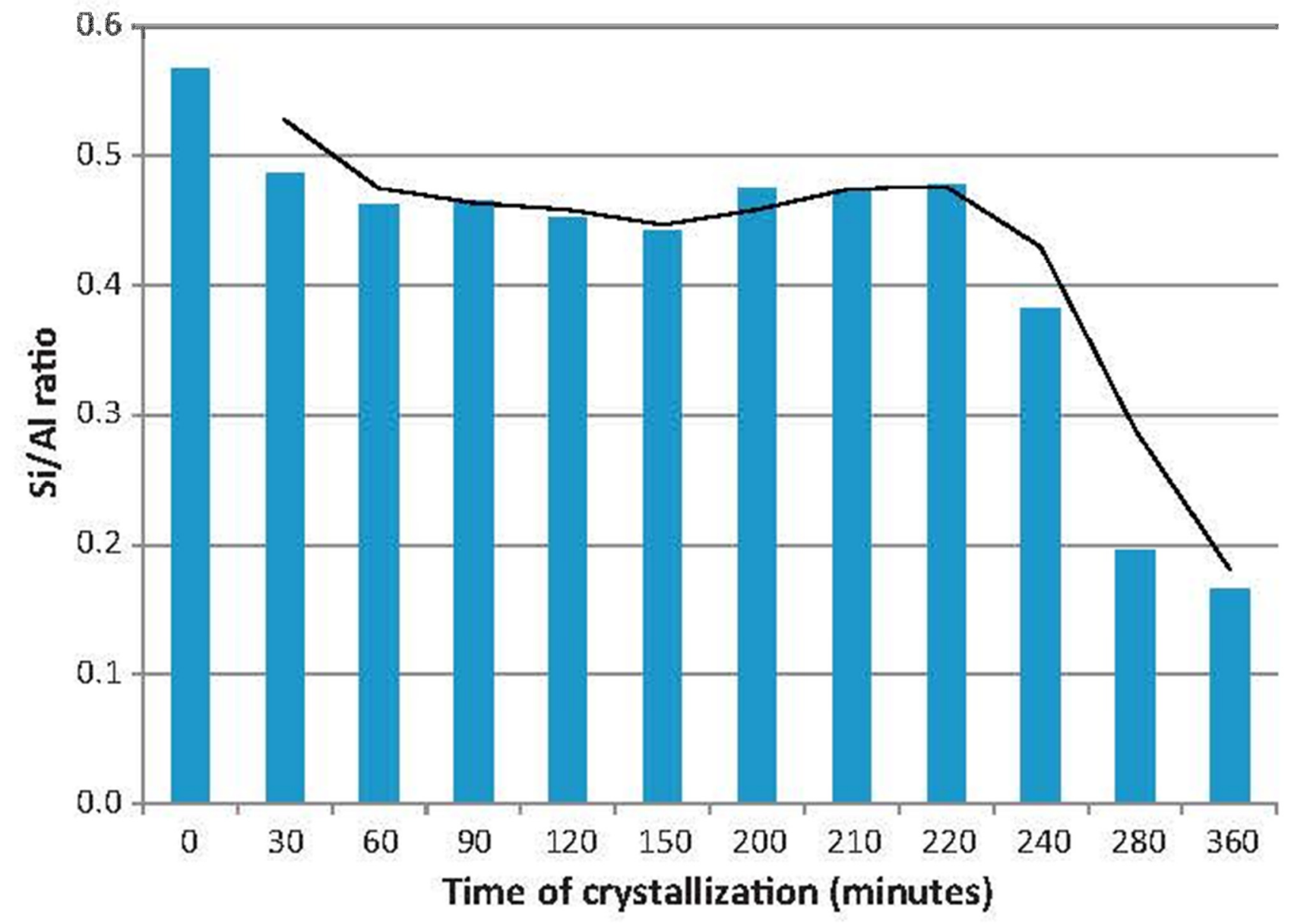

Fig. 12. ICP analysis: SUAI ratio of the supernatant solution for samples taken at

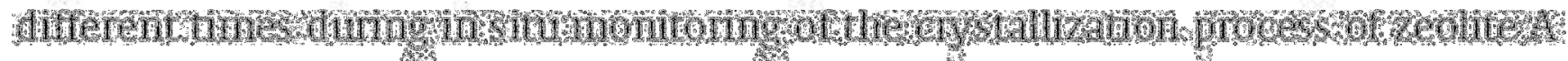

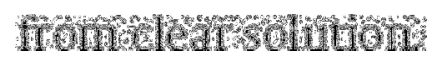

using clear solution. The assignment of IR bands for fully crystalline zeolite A synthesized after $360 \mathrm{~min}$ (at $80{ }^{\circ} \mathrm{C}$ ) in comparison to the standard zeolite A was straight forward and it gave the same characteristic bands at frequencies similar to the crystalline zeolite A synthesized from fly ash. The close matching of these IR bands indicated the similarity of their structural units and chemical moieties. From this observation, the IR technique can be concluded to be a good complementing technique to monitor development of crystallinity during the entire zeolite synthesis process.

\section{Discussion}

During the initial mixing of $\mathrm{Si}$ and $\mathrm{Al}$ source materials, the immediately formed product has been reported to be mostly non- equilibrated amorphous aluminosilicate gel $[29,30]$. This primary amorphous phase undergoes changes due to stirring and heating which in effect causes changes in reaction fluid properties such as viscosity and density. The reported observations can be seen from the fluctuations in the initial trend of in situ ultrasonic attenuation presented in Fig. 3 and further confirmed by the changes in the concentration of $\mathrm{Al}$ and Si shown in Figs. 10 and 11. 
Furthermore, Zhadanov [32] and Kerr [33] reported that equilibration with time of the initial amorphous gel with the liquid phase leads to the release of soluble species which is clearly noted by ICP analysis. Schmachtl [25] had also pointed out that during the initial stages of synthesis of aluminous zeolites structures such as LTA and FAU types, an amorphous aluminosilicate gel results after mixing reactive silica and aluminate source materials with alkali metal hydroxide ions in water. This observation can be confirmed by the presence of non-crystalline amorphous phase which acted as the precursor material prior to the crystallization of zeolite A as seen in the XRD results presented in Fig. 7. The slight increase of US- attenuation seen between 50 and $80 \mathrm{~min}$ in Fig. 3 deviates from the observation that was noted by Baser and Schwieger [28] who reported a continuative decrease of US-attenuation at the beginning of the hydrothermal synthesis process which they ascribed to the dissolution of the amorphous phase. This disruption of the US-attenuation trend can also be supported by Cundy and Cox's [29] concept of the formation of the 'pseudosteady-state intermediate' which they had referred to as 'secondary amorphous phase' occurring via solution transport mechanism during the process of evolution of order. The existence of primary (visible gel) and secondary amorphous phases had also been proposed by Angell and Flank [30]. During the nucleation period, between 120 and 150 min

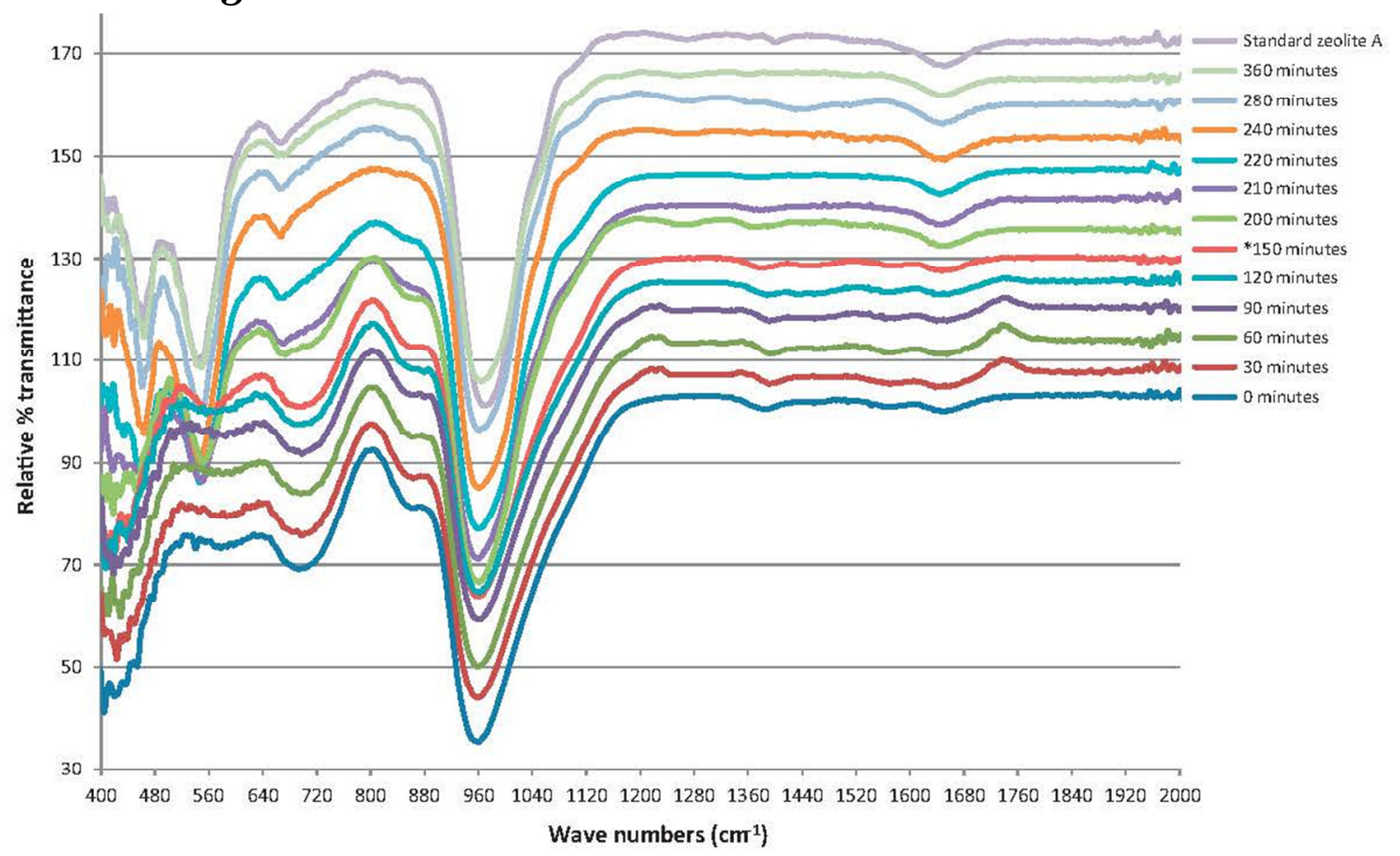

Fig. 13. FT-IR analysis of solid samples taken at different times during in situ monitoring of the crystallization process of zeolite A from clear solution with reference to standard zeolite A.

(see Fig. 3), complex rearrangements have been reported to take place leading to the formation of 'islands of order' which propagate with time and temperature (crystal growth) to form visible zeolite crystals [29]. The interface between solid and solvent during the nucleation stage has been reported to move faster [23] to an extent that it is not possible to notice significant changes in the concentration of $\mathrm{Al}$ and $\mathrm{Si}$ species in the solution (Figs. 10 and 11). This observation can further be confirmed by the negligible fluctuations occurring between 120 and $150 \mathrm{~min}$ in the in situ ultrasonic attenuation plot (Fig. 3). During this period, Pienack and Bensch [23] suggested that the reaction medium becomes supersaturated leading to the 
generation of a high free energy which is reduced by formation of a solid phase from the dissolved species.

The chemical and physical processes leading to formation of the nuclei as well as the duration of nucleation has been reported [29,34] to depend on the structure being formed and synthesis conditions. The acquisition of experimental data is very difficult during the nucleation process due to the extremely small nature of nuclei and only few a researchers have been successful in providing direct experimental evidence of nucleation phenomena [29,35-37]. The crystal growth period can be correlated to the steady increase of the US-attenuation occurring between 180 and 220 min in Fig. 3 and further confirmed by the increase in crystallinity of zeolite A as shown by XRD analysis in Fig. 7. This period is associated with systematic periodic propagation of zeolite crystals and can further be confirmed by the SEM images presented in Fig. 8 that show a reduction of the amorphous material as the size and number of zeolite A crystals increases. The growth rate of zeolite A crystals was reported to be around $37 \mathrm{~nm} / \mathrm{min}$ at near optimum compositions at around $80{ }^{\circ} \mathrm{C}$ [36]. The chemistry of development of order during the fundamental steps of crystal formation and growth has been discussed in depth by Cundy and Cox [29] as well as by Chang and Bell [35]. The S-shaped growth curve, in this case generated by measurements of US-attenuation against time, is commonly used to measure the zeolite crystallization kinetics [32]. Studies conducted by Miladinović [38] reported a 'stop effect' that was observed while carrying out in situ 27Al NMR monitoring of crystallization process of zeolite A. In this study, an almost similar observation was also observed by the use of in situ ultrasonic monitoring as the attenuation hump occur- ring at around 220 min in Fig. 3 and further confirmed by the drastic increase in the concentration of $\mathrm{Al}$ and $\mathrm{Si}$ at $220 \mathrm{~min}$ as shown in Figs. 10 and 11 respectively. This observation can be correlated to the breakdown of the amorphous gel structure. Similar observation was demonstrated by Mintova [34] using high resolution electron microscopy (HR-TEM) suggesting that the nucleation also occurred within the amorphous gel particles. The breakdown of the gel structure facilitated the release of nuclei 'germs' that was reported by Zhadanov [32] to lie dormant in the amorphous phase until activated by release into the solution. This observation is clearly seen by the drastic increase in the rate of crystallization as shown by the sharp increase in the ultrasonic attenuated signal occurring between 240 and 260 min in Fig. 3 and further demonstrated indirectly by the decrease in the concentration of $\mathrm{Al}$ and Si occurring in the same range as seen in Figs. 10 and 11. This unique behavior can also be related to the autocatalytic model of zeolite crystallization that was discussed by Walton [39]. Since there are two distinct inflection points observed during the crystallization monitoring process, we recommend independent calculation of kinetic parameters before and after the destruction of the gel structure since two S-shaped crystallization curves can be generated independently to mark the beginning and end of the attenuation decay. The second S-shaped curve (after gel structure destruction) can be generated by extrapolation. Kinetic studies of zeolite A crystallization from coal fly ash will be reported in an upcoming paper [50]. 
During the investigation of the ageing process, the initial decrease of the USattenuation can be associated with the temperature dependent influences on ultrasonic wave properties due to the exothermic nature of reactions taking place upon mixing fly ash extract with the aluminate solution. Partial dissolution is also thought to have occurred as can be shown by ICP analysis. The observations noted in this study support the effects of ageing which have been reported to results in acceleration of the overall crystallization process [32,40]. Cundy and Cox [29] suggested that ageing facilitates 'product-formation' effects such as introduction of reaction mixture entities 'nuclei' or increasing their number hence generating a different nucleation profile which would have otherwise not been the case if the reaction mixture followed a straight-forward (conventional) synthesis procedure. Other researchers [41] have concentrated on trying to generate direct experimental evidence to prove the conclusion that the ageing process enhances formation of viable nuclei even though it is not easy to get such evidence from chemical and spectroscopic studies. Modeling studies [42], kinetic analysis [43,44] and experiments based on analysis of ultimate product particle sizes as a function of different ageing times [41] have allowed interpretation of ageing-nucleation relationship and supported the conclusion. XRD analysis of the solid samples extracted before heating began (results not presented here) could not detect formation of any crystalline material. To answer the question why the 'hump' in the US-attenuation pattern disappeared in Fig. 5 during longer ageing time, the possibility of agglomeration of nuclei formed in the amorphous gel phase is thought to have occurred during the longer ageing periods hence minimizing the dramatic physico-chemical behavior that was associated with breakdown of the gel structure.

Comparing the formation process of zeolite A from fly ash precursors with that obtained from the simulated molar composition, the naked-eye milky observation after stirring and heating the simulated synthesis mixture was confirmed by the US-attenuation signal which can be seen as a peak in the US-attenuation pattern between 60 and 80 min (Fig. 6) signifying the formation of the secondary amorphous gel. The complex electrolyte solution generated from the coal fly ash as the starting material contains many different charged species whose concentrations and valence may complicate the understanding of the gel-solution-crystal interactions. This could be the reason why the hump in the US-attenuation that had been observed at around 220 min during the study of fly ash based gel was not observed in the attenuated pattern of the simulated composition. The presence of these additional ions in the fly ash-based reaction medium/mixture is expected to complicate the zeolitic crystal growth process by generating extra electrostatic contributions. Owing to the complexity of the starting composition, it is recommended that zeolite crystallization mechanism of each zeolite type synthesized from fly ash should be studied independently since it is not expected that the process will always follow the same reaction pathways. This suggestion is also supported by Walton [39,45] and Toufar [44] who had highlighted that each zeolite type follows a complex crystallization scheme even when using pure analytical grade sources of silica and alumina. The analysis of Si/Al ratio in Fig. 12 agreed well with studies by Miladinovic' [38] who had reported that an excess amount of 
Al leads to an increase of both the crystallization rate and crystal growth of zeolite A. The trend observed for $\mathrm{Si}$ and $\mathrm{Al}$ species in the extracted supernatant liquid compares very well with the ultrasonic signal that was obtained during the in situ monitoring process (Fig. 3) and can be used to explain the gel dissolution, equilibration, nucleation and consequent crystal growth processes which are key processes during the zeolitization process. The overall decrease in the ratio of the amount of amorphous material to the number of crystals of zeolite A shown in Fig. 8 is indicative and confirms the auto- catalytic process which suggest that the 'rate of gel dissolution must increase with the rate of consumption of growth species by increasing cumulative crystal surface area' [29,30,45].

When the US-attenuation plot obtained from synthesis using clear solution was compared to that obtained from the use of unseparated fly ash slurry (Fig. 4), it can be seen that the signal to noise ratio of the US-attenuation for the unseparated fly ash slurry was higher in relation to the use of clear solution which could be due to the presence of undissolved fused fly ash particles which caused scattering of the ultrasound waves. There are also differences in morphology of zeolite A crystals synthesized using clear solution with that from unseparated fly ash slurry, which can be attributed to the differences in the $\mathrm{Si} / \mathrm{Al}$ ratios during the respective synthesis process. The $\mathrm{Si} / \mathrm{Al}$ ratio of the raw unseparated reaction mixture is expected to change with time during synthesis process because more dissolution of the undissolved fly ash particles is expected to take place. Differences in morphology in relation to differences in $\mathrm{Si} / \mathrm{Al}$ ratios of different reaction compositions have also been reported by other researchers [46].

From infra-red analysis presented in Fig. 13, the amorphous aluminosilicate samples (o-120 min) showed a broad band centered at around $946 \mathrm{~cm}^{-1}$ that corresponded to $\mathrm{T} \quad \mathrm{O} \mathrm{T}$ (where $\mathrm{T}$ is either $\mathrm{Si}$ or $\mathrm{Al}$ that is tetrahedrally coordinated). This band shifted slightly and became shaper as the amorphous material was transformed into crystalline zeolites A. The IR band at $870 \mathrm{~cm}^{-1}$, which can be assigned to $\mathrm{T} \mathrm{OH}$ bond in the amorphous precursor of zeolite A, was also observed by Shigemoto [31]. Decottignies [47] noted that this band disappears as the sample became more crystalline. A general zeolite vibrational band assignment was summarized and reported by Flanigen [48]. The IR bands for the crystalline samples (150-360 min) were assigned as follows; IR band at $452 \mathrm{~cm}^{-1}$ can be assigned to $\mathrm{Si} \mathrm{Al} O$ bending mode [31], band at $660 \mathrm{~cm}^{-1}$ was assigned to $\mathrm{Si} \mathrm{Al} \mathrm{O}$ symmetric stretching while the band appearing $560 \mathrm{~cm}^{-1}$ has been related to the presence of the double ring (D4R) structure in the framework structure of zeolite A [49]. The band at $1640 \mathrm{~cm}^{-1}$ has been associated with the characteristic bending mode of water molecules. Comparing the spectra generated from amorphous sample (from o to $120 \mathrm{~min}$ ), the $1640 \mathrm{~cm}^{-1}$ band is not so prominent but becomes distinct for samples obtained after 150-360 min signifying that these samples had a higher percentage of water of hydration. Rayalu et al. [49] calculated the percentage crystallinity of zeolites by comparing the ratio of intensities of peaks at $560 \mathrm{~cm}^{-1}$ and to that appearing at 464 $\mathrm{cm}^{-1}$ (in this case 537 and $452 \mathrm{~cm}^{-1}$ respectively). 


\section{Conclusion}

The results presented herein have demonstrated clearly that the in situ ultrasonic diagnostic method can contribute significantly to a better understanding of the conversion process of coal fly ash to zeolites when compared with other studies conducted using other techniques reported in the literature. The findings from both the in situ ultrasonic monitoring system and the complementing ex situ techniques not only provide deeper insights into the reactions taking place during the conversion of coal fly ash to zeolites but also supported our proposal that formation of zeolite A from coal fly ash follows both the solution- and solid-phase mediated mechanisms for the zeolite formation. The solution mediated model is supported by the observation of small fluctuations of $\mathrm{Al}$ and Si concentration during nucleation and crystal growth step. Whereas solid-phase transformation growth model can be supported by the observation of the hump at around 220 min during the in situ ultrasonic monitoring (Fig. 3). This is complemented by an increase of Si and Al signifying the destruction of the amorphous gel structure which suggests that the amorphous phase is both the nutrient reservoir and also the host of the nucleation sites hence localized reconstruction of the gel particle to yield the dormant nucleation sites that are later released and activated.

\section{Acknowledgements}

The authors would like to acknowledge DAAD for funding, University of Erlangen Nürnberg, Germany for hosting Nicholas Musyoka during his DAAD scholarship period, Martin Hartman, Dieter Himsl, Albert Machoke and Regina Müller for help with SEM FT-IR, and ICP analysis. Hasan Baser and Wilhelm Schwieger gratefully acknowledge funding by the German Research Council (DFG), which supports the Cluster Excellence "Engineering of Advanced Materials" at the University of ErlangenNürnberg. 


\section{References}

[1] R.M. Barrer, Hydrothermal Chemistry of Zeolites, Academic Press, London, 1982.

[2] D.W. Breck, Zeolite Molecular Sieves, John Wiley \& Sons, New York, 1974.

[3] C. Wang, J. Li, L. Wang, X. Sun, Journal of Hazardous Materials 155 (2008) 5864.

[4] H. Tanaka, H. Eguchi, S. Fujimoto, R. Hino, Fuel 85 (2006) 1329-1334. 
[5] X. Querol, N. Moreno, J.C. Umana, A. Alastuey, E. Hernandez, A. Lopez-Soler, F. Plana, International Journal of Coal Geology 5o (2002) 413-423.

[6] M. Chareonpanich, O. Jullaphan, C. Tang, Journal of Cleaner Production 19 (2011) 58-63.

[7] V.S. Somerset, L.F. Petrik, R.A. White, M.J. Klink, D. Key, E. Iwuoha, Talanta 64 (2004) 109-114.

[8] V. Somerset, L. Petrik, E. Iwuoha, Journal of Environmental Management 87 (2008) 125-131.

[9] S. Chandrasekhar, P.N. Pramada, Microporous and Mesoporous Materials 108 (2008) 152-161.

[10] R. Anuwattana, P. Khummongkol, Journal of Hazardous Materials 166 (2009) 227-232.

[11] M. Sallam, Zeolite synthesis from municipal solid waste ash using fusion and hydrothermal treatment, PhD Thesis, University of South Florida, 2006.

[12] D.I. Petkowicz, R.T. Rigo, C. Radtke, S.B. Pergher, J.H.Z. dos Santos, Microporous and Mesoporous Materials 116 (2008) 548-554.

[13] H. Holler, H. Wirsching, Fortschritte der Mineralogie 63 (1985) 21-43.

[14] H. Chang, W. Shih, Industrial and Engineering Chemistry Research 39 (2000) 4185-4191.

[15] C. Baerlocher, L.B. McCusker, Database of Zeolite Structures (2008), http://www.iza-structure.org/databases/.

[16] K.S. Hui, C.Y.H. Chao, Journal of Hazardous Materials B 137 (2006) 401-409.

[17] Y. Liu, J. Xu, L. Jin, Y. Fang, H. Hu, Asia-Pacific Journal of Chemical Engineering 4 (2009) 666-671.

[18] K.S. Hui, C.Y.H. Chao, Environmental Science and Technology 42 (2008) $7392-7397$.

[19] G. Sankar, W. Bras, Catalysis Today 145 (2009) 195-203.

[20] J. Shi, M.W. Anderson, S.W. Carr, Chemistry of Materials 8 (1996) 369-375.

[21] F. Schüth, P. Bussian, P. Ågren, S. Schunk, M. Lindén, Solid State Sciences 3 
(2001) 801-808.

[22] Z. Miladinovic' , J. Zakrzewska, B. Kovačevic' , G. Bačic' , Materials Chemistry and Physics 104 (2007) 384-389.

[23] N. Pienack, N. Bensch, Angewandte Chemie International Edition 50 (2011) 2014-2034.

[24] H. Toufar, ESTEC study report, contract no. 12599/97/NL/NB, Noordwijk, 1998, 3-23.

[25] M. Schmachtl, T.J. Kim, W. Grill, R. Herrmann, O. Scharf, W. Schwieger, R. Schertlen, C. Stenzel, Ultrasonics 38 (2000) 809-812.

[26] R. Herrmann, W. Schwieger, O. Scharf, C. Stenzel, H. Toufar, M. Schmachtl, B. Ziberi, W. Grill, Microporous and Mesoporous Materials 80 (2005) 1-9.

[27] H. Baser, W. Schwieger, in: A. Gédéon, P. Massiani, F. Babonneau (Eds.), Zeo- lites and Related Materials: Trends, Targets and Challenges Proceedings of 4th International FEZA Conference, 2008, pp. 455-458.

[28] H. Baser, T. Selvam, J. Ofili, R. Herrmann, W. Schwieger, in: R. Xu, Z. Gao, J. Chen, W. Yan (Eds.), From Zeolites to Porous MOF Materials - The 40th Anniversary of International Zeolite Conference, 2007, pp. 480-486.

[29] C.S. Cundy, P.A. Cox, Microporous and Mesoporous Materials 82 (2005) $1-78$.

[30] C.L. Angell, W.H. Flank, in: J.R. Katzer (Ed.), Molecular Sieves-II, American Chemical Society Symposium Series, vol. 4O, 1977, p. 194.

[31] N. Shigemoto, S. Sugiyama, H. Hayashi, K. Miyaura, Journal of Materials Science 30 (1995) 5777.

[32] S.P. Zhadanov, in: E.M. Flanigen, L.B. San (Eds.), Molecular Sieve Zeolites1, American Chemical Society-Advances in Chemistry Series, vol. 101, 1971, p. 20.

[33] G.T. Kerr, Journal of Physical Chemistry 70 (1996) 1047.

[34] S. Mintova, N.H. Olson, J. Senker, T. Bein, Angewandte Chemie International Edition 41 (2002) 2558.

[35] C.D. Chang, A.T. Bell, Catalysis Letters 8 (1991) 305.

[36] L. Gora, K. Streletzky, R.W. Thompson, G.D.J. Phillies, Zeolites 18 (1997) 119. 
[37] C.S. Cundy, B.M. Lowe, D.M. Sinclair, Journal of the Chemical Society, Faraday 95 (1993) 235.

[38] Z. Miladinovic' , J. Zakrzewska, V. Dondur, Russian Journal of Physical Chemistry A 83 (2009) 1478-1484.

[39] R.I. Walton, F. Millange, D. O’Hare, A.T. Davies, G. Sankar, R.A. Catlow, Journal of Physical Chemistry B 105 (2001) 83.

[40] Q. Li, B. Mihailova, D. Creaser, J. Sterte, Microporous and Mesoporous Materials 43 (2001) 51.

[41] J.D. Cook, R.W. Thompson, Zeolites 8 (1988) 322.

[42] J. Bronic' , B. Subotic' , I. Šmit, Lj.A. Despotovic' , in: P.J. Grobet, W.J. Mortier, E.F. Vansant, G. Schulz-Ekloff (Eds.), Innovation in Zeolite Materials Science, Studies in Surface Science and Catalysis, vol. 37, Elsevier, Amsterdam, 1988, p. 107.

[43] A. Katovic' , B. Subotic' , I. Š mit, Lj.A. Despotovic' , M. C uric' , in: M.L. Occelli, H.E. Robson (Eds.), Zeolite Synthesis, American Chemical Society Symposium Series, vol. 398, 1989, p. 124.

[44] H. Toufar, K. Wendlandt, H.G. Karge, Journal of the Chemical Society, Faraday Transactions 91 (1995) 549-554.

[45] R.I. Walton, D. O'Hare, Journal of Physical Chemistry B 105 (2001) 91-96.

[46] E.I. Basaldella, A. Kikot, J.C. Tara, Materials Letters 31 (1997) 83-86.

[47] M. Decottignies, J. Phalippou, J. Zarzycki, Journal of Materials Science 13 (1978) 2605.

[48] E.M. Flanigen, H. Khatami, H.A. Szymanski, Advances in Chemistry Series $101(1971) 201$.

[49] S.S. Rayalu, J.S. Udhoji, S.U. Meshram, R.R. Naidu, S. Devotta, Current Science 89 (2005) 25.

[50] Unpublished results. 